\title{
Dividends and Dynamic Solvency Insurance in Two-Dimensional Risk Models
}

\author{
Cristina Gosio, Ester C. Lari, Marina Ravera, Maria-Laura Torrente \\ Department of Economics and Business Studies, University of Genoa, Genoa, Italy \\ Email: gosio@economia.unige.it, lari@economia.unige.it, ravera@economia.unige.it, marialaura.torrente@economia.unige.it
}

How to cite this paper: Gosio, C., Lari, E.C., Ravera, M. and Torrente, M.-L. (2018) Dividends and Dynamic Solvency Insurance in Two-Dimensional Risk Models. Modern Economy, 9, 2104-2118. https://doi.org/10.4236/me.2018.912131

Received: November 15, 2018 Accepted: December 10, 2018 Published: December 13, 2018

Copyright $\odot 2018$ by authors and Scientific Research Publishing Inc. This work is licensed under the Creative Commons Attribution International License (CC BY 4.0).

http://creativecommons.org/licenses/by/4.0/

(c) (i) Open Access

\begin{abstract}
In this paper we consider two-dimensional risk models where the claim counting processes of the two classes of business are assumed to be Poisson processes. We assume that the dividends are paid because of the presence of a reflecting upper barrier. Furthermore, in order to avoid ruin, we consider dynamic solvency insurance contracts that depend on two different definitions of time of ruin. We present a rather general model and, under different assumptions, we obtain the equations fulfilled by the discounted dividend payments and by the net single premium of dynamic solvency insurance. We also derive some boundary conditions and provide explicit solutions for some special cases.
\end{abstract}

\section{Keywords}

Two-Dimensional Risk Models, Dynamic Solvency Insurance, Dividends, Reflecting Barriers, Integro-Differential Equations

\section{Introduction}

The classical model of collective risk theory has been modified and extended over the years in several ways. Some authors [1] [2] [3] [4] have introduced a two-dimensional risk process where the insurer has two classes of business and in each of the two classes he has a surplus process similar to the one of the classical model. The two-dimensional risk process is defined by a two-dimensional vector having as components the above surplus processes. In this two-dimensional case, different interpretations of the concept of ruin have been proposed, and different definitions of time of ruin have been introduced. As it is well known, classical models of collective risk theory can forecast the dividends payment (for instance, see [5]) and, as proposed in [6], they can also have a dynamic solvency insurance. Under these assumptions we have models with two barriers. The 
two-dimensional models and some definitions of time of ruin encouraged us to investigate about dividends and the net single premium of a dynamic insurance contract in the two-dimensional models.

The paper is organized as follows: Section 2 is devoted to the presentation of the two-dimensional models under different claim arrival processes; Section 3 considers barriers referring to two definitions of time of ruin. Under these two different assumptions of time of ruin, Sections 4 and 5 contain the detailed computations to get the integro-differential equations, both of the net single premium of the dynamic solvency insurance and of the present value of dividends. In Sections 6 and 7 some boundary conditions for the above equations with some explicit solutions are given.

\section{The Model}

We assume that an insurer has two classes of business or insurance risks. Let $U_{i}(t)$ the surplus process, at time $t \geq 0$, of the $i$-th class given by:

$$
U_{i}(t)=u_{i}+c_{i} t-S_{i}(t)
$$

where:

- $u_{i}=U_{i}(0)$ is the initial surplus;

- $c_{i}>0$ is the constant premiun rate;

- $S_{i}(t)$ is the aggregate claim amount.

These assumptions define a two-dimensional risk model that can be formally stated as:

$$
\left(\begin{array}{l}
U_{1}(t) \\
U_{2}(t)
\end{array}\right)=\left(\begin{array}{l}
u_{1} \\
u_{2}
\end{array}\right)+\left(\begin{array}{l}
c_{1} \\
c_{2}
\end{array}\right) t-\left(\begin{array}{l}
S_{1}(t) \\
S_{2}(t)
\end{array}\right) .
$$

We assume that the claim number processes are

$$
\left\{N_{i}(t), i=1,2, t \geq 0\right\},
$$

which are defined as follows:

$$
N_{i}(t)=Q_{i}(t)+Q_{0}(t), i=1,2, t \geq 0,
$$

where $Q_{1}, Q_{2}$ and $Q_{0}$ are Poisson random variables with positive parameters $\theta_{1}, \theta_{2}$ and $\theta_{0}$, respectively.

Let $j$ be a strictly positive integer number and let $X_{i j}, i=1,2$, be the claim size random variables which we assume to have the same distribution function $F_{i}$ of class $C^{1}$, with:

- $F_{i}^{\prime}=f_{i}$, and such that $F_{i}(x)=0$ for $x \leq 0$.

Furthermore, we assume that:

- $E\left[X_{i j}\right]=\mu_{i}<+\infty$;

- the moment generating functions $M_{X_{i j}}(r)$ exist.

As usually stated, the random variables $X_{i j}$ are mutually independent for each $j=1,2, \cdots$, and are independent of $N_{i}(t), t \geq 0$.

Let $i=1,2$; the aggregate claim amount of each class of insurance risk is: 


$$
S_{i}(t)=\sum_{j=1}^{N_{i}(t)} X_{i j}
$$

Our two-dimensional risk model can be written as:

$$
\left(\begin{array}{l}
U_{1}(t) \\
U_{2}(t)
\end{array}\right)=\left(\begin{array}{l}
u_{1} \\
u_{2}
\end{array}\right)+\left(\begin{array}{l}
c_{1} \\
c_{2}
\end{array}\right) t-\left(\begin{array}{l}
\sum_{j=1}^{N_{1}(t)} X_{1 j} \\
\sum_{j=1}^{N_{2}(t)} X_{2 j}
\end{array}\right) .
$$

We observe that, since we have assumed $\theta_{0}, \theta_{1}$, and $\theta_{2}$ to be positive, the two counting processes $\left\{N_{1}(t)\right\}$ and $\left\{N_{2}(t)\right\}$, both appearing in (2), are correlated; a similar assumption has been made, for example, in [3].

If instead we assume $\theta_{0}>0$ and $\theta_{1}=\theta_{2}=0$, from (1) we have:

$$
N_{i}(t)=Q_{0}(t), i=1,2 \text {, }
$$

and the model (2) becomes:

$$
\left(\begin{array}{l}
U_{1}(t) \\
U_{2}(t)
\end{array}\right)=\left(\begin{array}{l}
u_{1} \\
u_{2}
\end{array}\right)+\left(\begin{array}{l}
c_{1} \\
c_{2}
\end{array}\right) t-\left(\begin{array}{c}
\sum_{j=1}^{Q_{0}(t)} X_{1 j} \\
\sum_{j=1}^{Q_{0}(t)} X_{2 j}
\end{array}\right),
$$

where the counting process $Q_{0}(t)$ is the same for both the classes of insurance risk (this assumption has been made, for instance, in [1] and in [2]).

Finally, if we assume $\theta_{0}=0$ and $\theta_{1}>0, \theta_{2}>0$, from (1) we get:

$$
N_{i}(t)=Q_{i}(t), i=1,2 \text {, }
$$

and the model (2) becomes:

$$
\left(\begin{array}{l}
U_{1}(t) \\
U_{2}(t)
\end{array}\right)=\left(\begin{array}{l}
u_{1} \\
u_{2}
\end{array}\right)+\left(\begin{array}{l}
c_{1} \\
c_{2}
\end{array}\right) t-\left(\begin{array}{c}
\sum_{j=1}^{Q_{1}(t)} X_{1 j} \\
\sum_{j=1}^{Q_{2}(t)} X_{2 j}
\end{array}\right),
$$

with different but independent counting processes for each class of insurance risk (this assumption has been made, for instance, in [4]).

\section{The Ruin and the Barriers}

It is well known that, in two-dimensional risk models, the time of ruin can be defined in different ways (see for instance [1] [2] and [3]):

$$
\begin{gathered}
T_{\text {sum }}=\inf \left\{t \mid U_{1}(t)+U_{2}(t)<0\right\} \\
T_{\text {min }}=\inf \left\{t \mid \min \left\{U_{1}(t), U_{2}(t)\right\}<0\right\}
\end{gathered}
$$

and

$$
T_{\text {max }}=\inf \left\{t \mid \max \left\{U_{1}(t), U_{2}(t)\right\}<0\right\} .
$$

In this paper we consider the time of ruin (7) or (8). Indeed, we observe that under the definition of $T_{\max }$ the ruin would not occur also in the case that one of the two surplus is deeply negative, provided that the other one is even only slightly positive: this scenario seems to be too risky for the insurance company.

Under the definition of $T_{\text {sum }}$ (7) the ruin does not occur when the surplus $U(t)=U_{1}(t)+U_{2}(t)$ is greater than or equal to zero; under the assumption (8) 
the survival occurs when both $U_{1}(t)$ and $U_{2}(t)$ are greater than or equal to zero. Therefore, the dynamic solvency insurance (see [6]) under the assumption (7) refers to the sum process and its payments are made immediately as soon as the surplus $U(t)$ falls below zero; instead, under the assumption (8), the dynamic solvency insurance refers separately to $U_{1}(t)$ and $U_{2}(t)$ and its payments are made immediately as soon as the surplus $U_{1}(t)$ or $U_{2}(t)$ falls below zero.

This means that, in the first scenario, we have a reflecting inferior barrier at zero for the sum process, whereas in the second scenario we have reflecting inferior barriers for each process with surplus $U_{i}(t), i=1,2$.

Even for the dividends payment it is necessary to introduce barriers. In particular, we assume to have the constant upper barrier $b_{i}$, with $b_{i} \geq u_{i}, i=1,2$, for the the $i$-th class of the two-dimensional risk process, and we consider to have the constant upper barrier $b=b_{1}+b_{2} \geq u_{1}+u_{2}$ for the sum process. We recall that dividends are paid whenever the corresponding surplus reaches its upper barrier.

Because of all the above assumptions, models in (2), (4) and (6) need to be consequently and coherently modified. This will be achieved in the following sections, where we address the problems of the net single premiums and the discounted dividend payments in the case of two barriers.

\section{The Net Single Premium of Dynamic Solvency Insurance}

This section is devoted to determine the equations fulfilled by the net single premium of the dynamic solvency insurance under the assumptions made in Sections 2 and 3. We use a procedure similar to the one presented in [7] and in [8] to determine the ruin probability. Hence, we introduce the force of interest $\delta>0$.

We first consider the case in which the time of ruin is $T_{\text {sum }}$ defined in (7). We therefore consider the sum process having a surplus with initial value (in $t=0) u=u_{1}+u_{2} \geq 0$ and constant premium rate $c=c_{1}+c_{2}>0$. The upper barrier is $b=b_{1}+b_{2} \geq u_{1}+u_{2}=u \geq 0$. Referring to the sum process, we recall that $U(t)=U_{1}(t)+U_{2}(t)$ is the surplus at time $t$, whose value is obviously dependent both on the dividends payment and on the assumption of the dynamic solvency insurance. We denote by $A(u, b)$ the net single premium of the dynamic solvency insurance. Let $T$ be the length of the interval time passing before the occurrence of some first claim for the sum process and let $X$ be the amount of the claim occurred at time $T$. If no claim occurs, we have that $U(t)=u+c t$ and $U(t)$ crosses the barrier $b$ at time $t^{*}$ defined by:

$$
t^{*}=\frac{b-u}{c} \text {. }
$$

Following the approach of [7] and [8], we compute $A(u, b)$ as the mean value of the random variable $\mathbf{A}(T, X)$. Because of the dividends payment, if $T=t$ and $X=x, \mathbf{A}(T, X)$ is given by: 


$$
\mathbf{A}(T, X)= \begin{cases}\mathrm{e}^{-\delta t} A(u+c t-x, b) & \text { if } t \leq t^{*} \text { and } x \leq u+c t \\ \mathrm{e}^{-\delta t}[A(0, b)+(x-(u+c t))] & \text { if } t \leq t^{*} \text { and } x>u+c t \\ \mathrm{e}^{-\delta t} A(b-x, b) & \text { if } t>t^{*} \text { and } x \leq b \\ \mathrm{e}^{-\delta t}[A(0, b)+(x-b)] & \text { if } t>t^{*} \text { and } x>b\end{cases}
$$

Since we have assumed that the arrival claims processes have the bivariate Poisson distribution defined in (1), the event $T=t$ happens when no claim occurs during the time interval going from 0 to $t$ and the first arrival of (any kind of) claim arrives at the end of this period of time.

Using the results of [9], the above events depend on the following probabilities (neglecting contributions of order higher than $\mathrm{d} t$ ):

$$
\begin{aligned}
& \quad \operatorname{Prob}\left[N_{1}(t)-N_{1}(0)=0 \text { and } N_{2}(t)-N_{2}(0)=0 \mid N_{1}(0)=N_{2}(0)=0\right] \\
& =\mathrm{e}^{-\left(\theta_{0}+\theta_{1}+\theta_{2}\right) t} \\
& \operatorname{Prob}\left[N_{1}(t+\mathrm{d} t)-N_{1}(t)=0 \text { and } N_{2}(t+\mathrm{d} t)-N_{2}(t)=0 \mid N_{1}(s), N_{2}(s), s \leq t\right] \\
& =\mathrm{e}^{-\left(\theta_{0}+\theta_{1}+\theta_{2}\right) \mathrm{d} t} \approx 1-\left(\theta_{0}+\theta_{1}+\theta_{2}\right) \mathrm{d} t \\
& \operatorname{Prob}\left[N_{1}(t+\mathrm{d} t)-N_{1}(t)=1 \text { and } N_{2}(t+\mathrm{d} t)-N_{2}(t)=0 \mid N_{1}(s), N_{2}(s), s \leq t\right] \\
& =\mathrm{e}^{-\left(\theta_{0}+\theta_{1}+\theta_{2}\right) \mathrm{d} t} \theta_{1} \mathrm{~d} t \approx\left(1-\left(\theta_{0}+\theta_{1}+\theta_{2}\right) \mathrm{d} t\right) \theta_{1} \mathrm{~d} t \approx \theta_{1} \mathrm{~d} t \\
& \operatorname{Prob}\left[N_{1}(t+\mathrm{d} t)-N_{1}(t)=0 \text { and } N_{2}(t+\mathrm{d} t)-N_{2}(t)=1 \mid N_{1}(s), N_{2}(s), s \leq t\right] \\
& =\mathrm{e}^{-\left(\theta_{0}+\theta_{1}+\theta_{2}\right) \mathrm{d} t} \theta_{2} \mathrm{~d} t \approx\left(1-\left(\theta_{0}+\theta_{1}+\theta_{2}\right) \mathrm{d} t\right) \theta_{2} \mathrm{~d} t \approx \theta_{2} \mathrm{~d} t \\
& \operatorname{Prob}\left[N_{1}(t+\mathrm{d} t)-N_{1}(t)=1 \text { and } N_{2}(t+\mathrm{d} t)-N_{2}(t)=1 \mid N_{1}(s), N_{2}(s), s \leq t\right] \\
& =\mathrm{e}^{-\left(\theta_{0}+\theta_{1}+\theta_{2}\right) \mathrm{d} t} \theta_{1} \theta_{2} \mathrm{~d} t^{2}\left(1+\frac{\theta_{0} \mathrm{~d} t}{\theta_{1} \theta_{2} \mathrm{~d} t^{2}}\right) \approx\left(1-\left(\theta_{0}+\theta_{1}+\theta_{2}\right) \mathrm{d} t\right) \theta_{0} \mathrm{~d} t \approx \theta_{0} \mathrm{~d} t
\end{aligned}
$$

Recalling that $T=t$ is the moment when some first claims occur, if we assume that at time $t$ it is $X_{11}=x_{1}$ and/or $X_{21}=x_{2}$ we have:

$$
X= \begin{cases}x_{1} & \text { with probability } \theta_{1} \mathrm{~d} t \\ x_{2} & \text { with probability } \theta_{2} \mathrm{~d} t \\ x_{1}+x_{2} & \text { with probability } \theta_{0} \mathrm{~d} t\end{cases}
$$

We denote by $F$ the distribution function of the random variable $X_{11}+X_{21}$. Because of the assumptions on the distribution functions $F_{i}, i=1,2$, it follows that (see [10])

$$
F(x)=\int_{0}^{x} F_{2}\left(x-x_{1}\right) f_{1}\left(x_{1}\right) \mathrm{d} x_{1},
$$

further, letting $F^{\prime}=f$ we have:

$$
f(x)=F^{\prime}(x)=\int_{0}^{x} f_{1}\left(x_{1}\right) f_{2}\left(x-x_{1}\right) \mathrm{d} x_{1} .
$$

Now, we are able to write the following integral equation for the net single premium of dynamic solvency insurance: 


$$
\begin{aligned}
& A(u, b)=E[\mathbf{A}(T, X)] \\
& =\int_{0}^{t^{*}} \mathrm{e}^{-\left(\theta_{0}+\theta_{1}+\theta_{2}\right) t}\left[\sum _ { i = 1 } ^ { 2 } \theta _ { i } \left(\int_{0}^{u+c t} \mathrm{e}^{-\delta t} A(u+c t-x, b) f_{i}(x) \mathrm{d} x\right.\right. \\
& \left.\quad+\int_{u+c t}^{+\infty} \mathrm{e}^{-\delta t}(A(0, b)+(x-(u+c t))) f_{i}(x) \mathrm{d} x\right) \\
& +\theta_{0}\left(\int_{0}^{u+c t} \mathrm{e}^{-\delta t} A(u+c t-x, b) f(x) \mathrm{d} x\right. \\
& \left.\left.+\int_{u+c t}^{+\infty} \mathrm{e}^{-\delta t}(A(0, b)+(x-(u+c t))) f(x) \mathrm{d} x\right)\right] \mathrm{d} t \\
& +\int_{t^{*}}^{+\infty} \mathrm{e}^{-\left(\theta_{0}+\theta_{1}+\theta_{2}\right) t}\left[\sum _ { i = 1 } ^ { 2 } \theta _ { i } \left(\int_{0}^{b} \mathrm{e}^{-\delta t} A(b-x, b) f_{i}(x) \mathrm{d} x\right.\right. \\
& \left.+\int_{b}^{+\infty} \mathrm{e}^{-\delta t}(A(0, b)+(x-b)) f_{i}(x) \mathrm{d} x\right) \\
& +\theta_{0}\left(\int_{0}^{b} \mathrm{e}^{-\delta t} A(b-x, b) f(x) \mathrm{d} x\right. \\
& \left.\left.+\int_{b}^{+\infty} \mathrm{e}^{-\delta t}(A(0, b)+(x-b)) f(x) \mathrm{d} x\right)\right] \mathrm{d} t
\end{aligned}
$$

Hence, by substituting $u+c t$ with $z$, and subsequently $b-x$ with $y$, we obtain:

$$
\begin{aligned}
A(u, b)= & \frac{1}{c} \mathrm{e}^{\left(\theta_{0}+\theta_{1}+\theta_{2}+\delta\right) \frac{u}{c}}\left\{\int _ { u } ^ { b } \mathrm { e } ^ { - ( \theta _ { 0 } + \theta _ { 1 } + \theta _ { 2 } + \delta ) \frac { z } { c } } \left[\sum _ { i = 1 } ^ { 2 } \theta _ { i } \left(\int_{0}^{z} A(z-x, b) f_{i}(x) \mathrm{d} x\right.\right.\right. \\
& \left.+\int_{z}^{+\infty}(A(0, b)+(x-z)) f_{i}(x) \mathrm{d} x\right)+\theta_{0}\left(\int_{0}^{z} A(z-x, b) f(x) \mathrm{d} x\right. \\
& \left.\left.+\int_{z}^{+\infty}(A(0, b)+(x-z)) f(x) \mathrm{d} x\right)\right] \mathrm{d} z+\int_{b}^{+\infty} \mathrm{e}^{-\left(\theta_{0}+\theta_{1}+\theta_{2}+\delta\right) \frac{z}{c}} \\
& \cdot\left[\sum_{i=1}^{2} \theta_{i} \int_{0}^{b}(A(y, b)+y) f_{i}(b-y) \mathrm{d} y-\sum_{i=1}^{2} \theta_{i} b F_{i}(b)+\sum_{i=1}^{2}\left(\theta_{i}+\theta_{0}\right) \mu_{i}\right. \\
& +(A(0, b)-b)\left(\sum_{i=1}^{2} \theta_{i}\left(1-F_{i}(b)\right)+\theta_{0}(1-F(b))\right) \\
& \left.\left.+\theta_{0} \int_{0}^{b}(A(y, b)+y) f(b-y) \mathrm{d} y-\theta_{0} b F(b)\right] \mathrm{d} z\right\}
\end{aligned}
$$

By deriving (12) with respect to $u$, we obtain the following integro-differential equation:

$$
\begin{aligned}
& c \frac{\partial A(u, b)}{\partial u}-\left(\theta_{0}+\theta_{1}+\theta_{2}+\delta\right) A(u, b)+\sum_{i=1}^{2}\left(\theta_{i}+\theta_{0}\right) \mu_{i} \\
& +(A(0, b)-u)\left[\sum_{i=1}^{2} \theta_{i}\left(1-F_{i}(u)\right)+\theta_{0}(1-F(u))\right] \\
& =-\sum_{i=1}^{2} \theta_{i} \int_{0}^{u}(A(y, b)+y) f_{i}(u-y) \mathrm{d} y+\sum_{i=1}^{2} \theta_{i} u F_{i}(u) \\
& \quad-\theta_{0} \int_{0}^{u}(A(y, b)+y) f(u-y) \mathrm{d} y+\theta_{0} u F(u)
\end{aligned}
$$

Obviously, if $\theta_{1}=\theta_{2}=0$, expression (13) leads to the equation of the net single premium of the solvency insurance for the model given in (4). Similarly, if $\theta_{0}=0$, from (13) we find the equation of the net single premium of the solvency insurance contract for the model given in (6).

We now consider the case in which the time of ruin is $T_{\min }$ defined by (8). 
We recall that, using this definition, the ruin occurs when at least one of the surplus $U_{i}(t), i=1,2$, becomes negative. Thus, the dynamic solvency insurance has to intervene separately in the two classes, and it has to avoid that both surplus become negative. Let $i=1,2$ and let $A_{i}\left(u_{i}, b_{i}\right)$ be the net single premium of the dynamic solvency insurance contract of the $i$-th insurance class. The two-dimensional risk process holder will have to bear the total single premium $\sum_{i=1}^{2} A_{i}\left(u_{i}, b_{i}\right)$. In the following, using a procedure similar to the one adopted in the previous discussion, we deduce the equations fulfilled by $A_{i}\left(u_{i}, b_{i}\right)$, with $i=1,2$. To this end, we let $T^{i}$ be the length of the interval time passing before the occurrence of some first claim for the $i$-th process and $X_{i 1}$ be the amount of the first claim. If no claim occurs, we have that $U_{i}(t)=u_{i}+c_{i} t$ and $U_{i}(t)$ crosses the barrier $b_{i}$ at time $t_{i}^{*}$ defined by:

$$
t_{i}^{*}=\frac{b_{i}-u_{i}}{c_{i}} .
$$

We compute $A_{i}\left(u_{i}, b_{i}\right)$ as the mean value of the random variable $\mathbf{A}_{i}\left(T^{i}, X_{i 1}\right)$. Because of the payment of the dividends, in the case $T^{i}=t_{i}$ and $X_{i 1}=x_{i}$, arguing as in the case of the sum process, $\mathbf{A}_{i}\left(T^{i}, X_{i 1}\right)$ can be simply obtained by evaluating (10) at $A=A_{i}, u=u_{i}, c=c_{i}, b=b_{i}, t^{*}=t_{i}^{*}$ defined in (14). We recall that the arrival claims process has the bivariate Poisson distribution defined in (1). Since the two processes are considered separately, for any $i=1,2$ it results (neglecting contributions of order higher than $\mathrm{d} t$ ):

$$
\begin{aligned}
& \operatorname{Prob}\left[N_{i}(t)-N_{i}(0)=0 \mid N_{i}(0)=0\right]=\mathrm{e}^{-\left(\theta_{0}+\theta_{i}\right) t} \\
& \operatorname{Prob}\left[N_{i}(t+\mathrm{d} t)-N_{i}(t)=0 \mid N_{i}(s), s \leq t\right] \\
& =\mathrm{e}^{-\left(\theta_{0}+\theta_{i}\right) \mathrm{d} t} \approx\left(1-\left(\theta_{0}+\theta_{i}\right) \mathrm{d} t\right) \\
& \operatorname{Prob}\left[N_{i}(t+\mathrm{d} t)-N_{i}(t)=0 \mid N_{i}(s), s \leq t\right] \\
& =\mathrm{e}^{-\left(\theta_{0}+\theta_{i}\right) \mathrm{d} t} \approx\left(1-\left(\theta_{0}+\theta_{i}\right) \mathrm{d} t\right)
\end{aligned}
$$

Hence, recalling that $T^{i}=t_{i}$ is the moment when some first claims occur and that the amount of the claim is $X_{i 1}$ with $X_{i 1}=x_{i}$, it results:

$$
X_{i 1}=x_{i} \text { with probability }\left(\theta_{0}+\theta_{i}\right) \mathrm{d} t \text {. }
$$

We are now able to write the following integral equation for the net single premium of dynamic solvency insurance for the $i$-th claim, $i=1,2$ :

$$
\begin{aligned}
& A_{i}\left(u_{i}, b_{i}\right)=E\left[\mathbf{A}_{i}\left(T^{i}, X_{i 1}\right)\right] \\
& =\int_{0}^{t_{i}^{*}} \mathrm{e}^{-\left(\theta_{0}+\theta_{i}\right) t}\left(\theta_{0}+\theta_{i}\right)\left[\int_{0}^{u_{i}+c_{i} t} \mathrm{e}^{-\delta t} A_{i}\left(u_{i}+c_{i} t-x, b_{i}\right) f_{i}(x) \mathrm{d} x\right. \\
& \left.+\int_{u_{i}+c_{i} t}^{+\infty} \mathrm{e}^{-\delta t}\left(A_{i}\left(0, b_{i}\right)+\left(x-\left(u_{i}+c_{i} t\right)\right)\right) f_{i}(x) \mathrm{d} x\right] \mathrm{d} t \\
& +\int_{t_{i}^{*}}^{+\infty} \mathrm{e}^{-\left(\theta_{0}+\theta_{i}\right) t}\left(\theta_{0}+\theta_{i}\right)\left[\int_{0}^{b_{i}} \mathrm{e}^{-\delta t} A_{i}\left(b_{i}-x, b_{i}\right) f_{i}(x) \mathrm{d} x\right. \\
& \left.+\int_{b_{i}}^{+\infty} \mathrm{e}^{-\delta t}\left(A_{i}\left(0, b_{i}\right)+\left(x-b_{i}\right)\right) f_{i}(x) \mathrm{d} x\right] \mathrm{d} t
\end{aligned}
$$

Hence, by substituting $u_{i}+c_{i} t$ with $z$, and subsequently $b_{i}-x$ with $y$, we obtain: 


$$
\begin{aligned}
& A_{i}\left(u_{i}, b_{i}\right) \\
& =\frac{\theta_{0}+\theta_{i}}{c_{i}} \mathrm{e}^{\left(\theta_{0}+\theta_{i}+\delta\right) \frac{u_{i}}{c_{i}}}\left\{\int _ { u _ { i } } ^ { b _ { i } } \mathrm { e } ^ { - ( \theta _ { 0 } + \theta _ { i } + \delta ) \frac { z } { c _ { i } } } \left[\int_{0}^{z}\left(A_{i}\left(z-x, b_{i}\right)-x\right) f_{i}(x) \mathrm{d} x\right.\right. \\
& \left.+\left(A_{i}\left(0, b_{i}\right)-z\right)\left(1-F_{i}(z)\right)+\mu_{i}\right] \mathrm{d} z \\
& +\int_{b_{i}}^{+\infty} \mathrm{e}^{-\left(\theta_{0}+\theta_{i}+\delta\right) \frac{z}{c_{i}}}\left[\int_{0}^{b_{i}}\left(A_{i}\left(y, b_{i}\right)+y\right) f_{i}\left(b_{i}-y\right) \mathrm{d} y-b_{i} F_{i}\left(b_{i}\right)\right. \\
& \left.\left.+\left(A_{i}\left(0, b_{i}\right)-b_{i}\right)\left(1-F_{i}\left(b_{i}\right)\right)+\mu_{i}\right] \mathrm{~d} z\right\}, i=1,2
\end{aligned}
$$

By deriving (16) with respect to $u_{i}$, we obtain the following integro-differential equation:

$$
\begin{aligned}
& c_{i} \frac{\partial A_{i}\left(u_{i}, b_{i}\right)}{\partial u_{i}}-\left(\theta_{0}+\theta_{i}+\delta\right) A_{i}\left(u_{i}, b_{i}\right)+\left(\theta_{0}+\theta_{i}\right) \mu_{i} \\
& +\left(\theta_{0}+\theta_{i}\right)\left(A_{i}\left(0, b_{i}\right)-u_{i}\right)\left(1-F_{i}\left(u_{i}\right)\right) \\
& =-\left(\theta_{0}+\theta_{i}\right) \int_{0}^{u_{i}}\left(A_{i}\left(y, b_{i}\right)+y\right) f_{i}\left(u_{i}-y\right) \mathrm{d} y+\left(\theta_{0}+\theta_{i}\right) u_{i} F_{i}\left(u_{i}\right), i=1,2
\end{aligned}
$$

Obviously, if we restrict ourselves to the case of model given in (4), that is if we let $\theta_{1}=\theta_{2}=0$, expressions (16) and (17) lead to the same kind of equation for the net single premium of the two classes of insurance business. In the same vein, if we consider the model given in (6), that is if we let $\theta_{0}=0$, Equations (12)-(13) and (16)-(17) are comparable.

\section{Discounted Value of the Dividend Payments}

In this section we determine the equations fulfilled by the discounted value of the dividend payments under the different assumptions made in Section 4. To get our results, we follow a similar approach to the one used for the net single premium of dynamic solvency insurance.

We first consider the case where the time of ruin is $T_{\text {sum }}$ defined in (7). Hence, we consider the sum process having a surplus $U(t)$ with initial value (in $t=0$ ) $u=u_{1}+u_{2} \geq 0$ and constant premium rate $c=c_{1}+c_{2}>0$. The upper barrier is $b=b_{1}+b_{2} \geq 0$. We let $T$ be the length of the interval time passing before the occurrence of some first claim for the sum process and $X$ be the amount of the claim occurred in $T$. If no claims occur, then $U(t)$ crosses the barrier $b$ at time $t^{*}$ defined in (9). We denote by $D(u, b)$ the discounted value of the dividend payments, using the force of interest $\delta>0$.

We find $D(u, b)$ as the mean value of the random variable $\mathbf{D}(T, X)$. Because of the dynamic solvency insurance, in the case $T=t$ and $X=x$, $\mathbf{D}(T, X)$ is given by:

$$
\mathbf{D}(T, X)= \begin{cases}\mathrm{e}^{-\delta t} D(u+c t-x, b) & \text { if } t \leq t^{*} \text { and } x \leq u+c t \\ \mathrm{e}^{-\delta t} D(0, b) & \text { if } t \leq t^{*} \text { and } x>u+c t \\ \mathrm{e}^{-\delta t} D(b-x, b)+\mathcal{D}(u, b, t) & \text { if } t>t^{*} \text { and } x \leq b \\ \mathrm{e}^{-\delta t} D(0, b)+\mathcal{D}(u, b, t) & \text { if } t>t^{*} \text { and } x>b\end{cases}
$$

where $\mathcal{D}(u, b, t)$ is the value of the dividends paid in the interval time $\left(t^{*}, t\right)$, evaluated at $t=0$ : 


$$
\mathcal{D}(u, b, t)=\int_{t^{*}}^{t} c \mathrm{e}^{-\delta s} \mathrm{~d} s=c \frac{\mathrm{e}^{-\delta t^{*}}-\mathrm{e}^{-\delta t}}{\delta} .
$$

As in Section 4, we assume that $T=t$ is the instant when claims occur. If at time $t$ we have $X_{11}=x_{1}$ and/or $X_{21}=x_{2}$, then formula (11) still holds. Therefore, we are able to write the following integral equation for the discounted value of the dividend payments:

$$
\begin{aligned}
& D(u, b)=E[\mathbf{D}(T, X)] \\
& =\int_{0}^{t^{*}} \mathrm{e}^{-\left(\theta_{0}+\theta_{1}+\theta_{2}\right) t}\left[\sum _ { i = 1 } ^ { 2 } \theta _ { i } \left(\int_{0}^{u+c t} \mathrm{e}^{-\delta t} D(u+c t-x, b) f_{i}(x) \mathrm{d} x\right.\right. \\
& \left.\quad+\int_{u+c t}^{+\infty} \mathrm{e}^{-\delta t} D(0, b) f_{i}(x) \mathrm{d} x\right)+\theta_{0}\left(\int_{0}^{u+c t} \mathrm{e}^{-\delta t} D(u+c t-x, b) f(x) \mathrm{d} x\right. \\
& \left.\left.\quad+\int_{u+c t}^{+\infty} \mathrm{e}^{-\delta t} D(0, b) f(x) \mathrm{d} x\right)\right] \mathrm{d} t+\int_{t^{*}}^{+\infty} \mathrm{e}^{-\left(\theta_{0}+\theta_{1}+\theta_{2}\right) t} \\
& +\left[\sum_{i=1}^{2} \theta_{i}\left(\int_{0}^{b} \mathrm{e}^{-\delta t} D(b-x, b) f_{i}(x) \mathrm{d} x+\int_{b}^{+\infty} \mathrm{e}^{-\delta t} D(0, b) f_{i}(x) \mathrm{d} x\right)\right. \\
& \left.+\theta_{0}\left(\int_{0}^{b} \mathrm{e}^{-\delta t} D(b-x, b) f(x) \mathrm{d} x+\int_{b}^{+\infty} \mathrm{e}^{-\delta t} D(0, b) f(x) \mathrm{d} x\right)\right] \mathrm{d} t \\
& +\int_{t^{*}}^{+\infty} \mathrm{e}^{-\left(\theta_{0}+\theta_{1}+\theta_{2}\right) t}\left[\sum_{i=1}^{2} \theta_{i} \int_{0}^{+\infty} \mathcal{D}(u, b, t) f_{i}(x) \mathrm{d} x\right. \\
& \left.+\theta_{0} \int_{0}^{+\infty} \mathcal{D}(u, b, t) f(x) \mathrm{d} x\right] \mathrm{d} t
\end{aligned}
$$

where, by using (19) and with some computations, the last integral can be rewritten as follows:

$$
\begin{aligned}
& \int_{t^{*}}^{+\infty} \mathrm{e}^{-\left(\theta_{0}+\theta_{1}+\theta_{2}\right) t}\left[\sum_{i=1}^{2} \theta_{i} \int_{0}^{+\infty} \mathcal{D}(u, b, t) f_{i}(x) \mathrm{d} x+\theta_{0} \int_{0}^{+\infty} \mathcal{D}(u, b, t) f(x) \mathrm{d} x\right] \mathrm{d} t \\
& =\int_{t^{*}}^{+\infty} \mathrm{e}^{-\left(\theta_{0}+\theta_{1}+\theta_{2}\right) t}\left(\theta_{0}+\theta_{1}+\theta_{2}\right) \mathcal{D}(u, b, t) \mathrm{d} t \\
& =\frac{c}{\theta_{0}+\theta_{1}+\theta_{2}+\delta} \mathrm{e}^{-\left(\theta_{0}+\theta_{1}+\theta_{2}+\delta\right)\left(\frac{b}{c}-\frac{u}{c}\right)}
\end{aligned}
$$

Hence, by substituting in (20) the expression (21) and the new variables $z=u+c t$ and $y=b-x$, we obtain:

$$
\begin{aligned}
D & (u, b) \\
= & \mathrm{e}^{\left(\theta_{0}+\theta_{1}+\theta_{2}+\delta\right) \frac{u}{c}}\left\{\frac { 1 } { c } \int _ { u } ^ { b } \mathrm { e } ^ { - ( \theta _ { 0 } + \theta _ { 1 } + \theta _ { 2 } + \delta ) \frac { z } { c } } \left[\sum _ { i = 1 } ^ { 2 } \theta _ { i } \left(\int_{0}^{z} D(z-x, b) f_{i}(x) \mathrm{d} x\right.\right.\right. \\
& \left.+\int_{z}^{+\infty} D(0, b) f_{i}(x) \mathrm{d} x\right)+\theta_{0}\left(\int_{0}^{z} D(z-x, b) f(x) \mathrm{d} x\right. \\
& \left.\left.+\int_{z}^{+\infty} D(0, b) f(x) \mathrm{d} x\right)\right] \mathrm{d} z+\frac{1}{c} \int_{b}^{+\infty} \mathrm{e}^{-\left(\theta_{0}+\theta_{1}+\theta_{2}+\delta\right) \frac{z}{c}} \\
& +\left[\sum_{i=1}^{2} \theta_{i} \int_{0}^{b} D(y, b) f_{i}(b-y) \mathrm{d} y+\sum_{i=1}^{2} \theta_{i}\left(1-F_{i}(b)\right) D(0, b)\right. \\
& \left.+\theta_{0} \int_{0}^{b} D(y, b) f(b-y) \mathrm{d} y+\theta_{0}(1-F(b)) D(0, b)\right] \mathrm{d} z \\
& \left.+\frac{c}{\theta_{0}+\theta_{1}+\theta_{2}+\delta} \mathrm{e}^{-\left(\theta_{0}+\theta_{1}+\theta_{2}+\delta\right) \frac{b}{c}}\right\}
\end{aligned}
$$

From the previous expression, deriving with respect to $u$, we obtain the following integro-differential equation: 


$$
\begin{aligned}
& c \frac{\partial D(u, b)}{\partial u}-\left(\theta_{0}+\theta_{1}+\theta_{2}+\delta\right) D(u, b) \\
& +\sum_{i=1}^{2} \theta_{i}\left(1-F_{i}(u)\right) D(0, b)+\theta_{0}(1-F(u)) D(0, b) \\
& =-\sum_{i=1}^{2} \theta_{i} \int_{0}^{u} D(y, b) f_{i}(u-y) \mathrm{d} y-\theta_{0} \int_{0}^{u} D(y, b) f(u-y) \mathrm{d} y
\end{aligned}
$$

Obviously, letting $\theta_{1}=\theta_{2}=0$ in (23), we find the equation of the discounted value of the dividend payments of the solvency insurance for the model given in (23). Similarly, if $\theta_{0}=0$, from (23) we find the equation of the discounted value of the dividend payments for the model given in (6).

We now consider the case in which the time of ruin is $T_{\min }$ defined by (8). We note that the observations pointed out in Section 4 still hold and we determine the equations fulfilled by the present value of the dividend payments. We let $i=1,2$; we denote by $D_{i}\left(u_{i}, b_{i}\right)$ the present value of the dividend payments of the $i$-th insurance class. The total dividends of the two-dimensional risk process holder will be equal to $\sum_{i=1}^{2} D_{i}\left(u_{i}, b_{i}\right)$. Even in this case, we find $D_{i}\left(u_{i}, b_{i}\right)$ as the mean value of the random variable $\mathbf{D}_{i}\left(T^{i}, X_{i 1}\right)$ that, because of the payment of the dividends, if $T^{i}=t_{i}$ and $X_{i 1}=x_{i}$, can be simply obtained by evaluating (18) and (19) at $D=D_{i}, u=u_{i}, c=c_{i}, \quad b=b_{i}$, and $t^{*}=t_{i}^{*}$ defined in (14). Following Section 4, we note that formula (15) still holds. Therefore, we are able to write the following integral equation for the discounted value of the dividend payments:

$$
\begin{aligned}
& D_{i}\left(u_{i}, b_{i}\right)=E\left[\mathbf{D}_{i}\left(T^{i}, X_{i 1}\right)\right] \\
& =\int_{0}^{t_{i}^{*}} \mathrm{e}^{-\left(\theta_{0}+\theta_{i}\right) t}\left(\theta_{0}+\theta_{i}\right)\left[\int_{0}^{u_{i}+c_{i} t} \mathrm{e}^{-\delta t} D_{i}\left(u_{i}+c_{i} t-x, b_{i}\right) f_{i}(x) \mathrm{d} x\right. \\
& \left.\quad+\int_{u_{i}+c_{i} t}^{+\infty} \mathrm{e}^{-\delta t} D_{i}\left(0, b_{i}\right) f_{i}(x) \mathrm{d} x\right] \mathrm{d} t+\int_{t_{i}^{*}}^{+\infty} \mathrm{e}^{-\left(\theta_{0}+\theta_{i}\right) t}\left(\theta_{0}+\theta_{i}\right) \\
& \quad \cdot\left[\int_{0}^{b_{i}} \mathrm{e}^{-\delta t} D_{i}\left(b_{i}-x, b_{i}\right) f_{i}(x) \mathrm{d} x+\int_{b_{i}}^{+\infty} \mathrm{e}^{-\delta t} D_{i}\left(0, b_{i}\right) f_{i}(x) \mathrm{d} x\right] \mathrm{d} t \\
& \quad+\int_{t_{i}^{*}}^{+\infty} \mathrm{e}^{-\left(\theta_{0}+\theta_{i}\right) t}\left(\theta_{0}+\theta_{i}\right) \mathcal{D}\left(u_{i}, b_{i}, t\right) \mathrm{d} t
\end{aligned}
$$

where, by using (19) and with some computations, the last integral can be rewritten as follows:

$$
\int_{t_{i}^{*}}^{+\infty} \mathrm{e}^{-\left(\theta_{0}+\theta_{i}\right) t}\left(\theta_{0}+\theta_{i}\right) \mathcal{D}\left(u_{i}, b_{i}, t\right) \mathrm{d} t=\frac{c_{i}}{\theta_{0}+\theta_{i}+\delta} \mathrm{e}^{-\left(\theta_{0}+\theta_{i}+\delta\right)\left(\frac{b_{i}}{c_{i}}-\frac{u_{i}}{c_{i}}\right)} .
$$

Hence, by substituting in (24) the expression (25) and the new variables $z=u_{i}+c_{i} t$ and $y=b_{i}-x$, we get:

$$
\begin{aligned}
& D_{i}\left(u_{i}, b_{i}\right) \\
& =\frac{\theta_{0}+\theta_{i}}{c_{i}} \mathrm{e}^{\left(\theta_{0}+\theta_{i}+\delta\right) \frac{u_{i}}{c_{i}}}\left\{\int _ { u _ { i } } ^ { b _ { i } } \mathrm { e } ^ { - ( \theta _ { 0 } + \theta _ { i } + \delta ) \frac { z } { c _ { i } } } \left[\int_{0}^{z} D_{i}\left(z-x, b_{i}\right) f_{i}(x) \mathrm{d} x\right.\right. \\
& \left.+\int_{z}^{+\infty} D_{i}\left(0, b_{i}\right) f_{i}(x) \mathrm{d} x\right] \mathrm{d} z+\int_{b_{i}}^{+\infty} \mathrm{e}^{-\left(\theta_{0}+\theta_{i}+\delta\right) \frac{z}{c_{i}}}\left[\int_{0}^{b_{i}} D_{i}\left(y, b_{i}\right) f_{i}\left(b_{i}-y\right) \mathrm{d} y\right. \\
& \left.\left.+D_{i}\left(0, b_{i}\right)\left(1-F_{i}\left(b_{i}\right)\right)\right] \mathrm{d} z\right\}+c_{i} \frac{\mathrm{e}^{-\left(\theta_{0}+\theta_{i}+\delta\right)}\left(\frac{b_{i}}{c_{i}}-\frac{u_{i}}{c_{i}}\right)}{\theta_{0}+\theta_{i}+\delta}, i=1,2
\end{aligned}
$$


From the previous expression, deriving with respect to $u_{i}$, we obtain the following integro-differential equation:

$$
\begin{aligned}
& c_{i} \frac{\partial D_{i}\left(u_{i}, b_{i}\right)}{\partial u_{i}}-\left(\theta_{0}+\theta_{i}+\delta\right) D_{i}\left(u_{i}, b_{i}\right)+\left(\theta_{0}+\theta_{i}\right) D_{i}\left(0, b_{i}\right)\left(1-F_{i}\left(u_{i}\right)\right) \\
& =-\left(\theta_{0}+\theta_{i}\right) \int_{0}^{u_{i}} D_{i}\left(y, b_{i}\right) f_{i}\left(u_{i}-y\right) \mathrm{d} y, i=1,2
\end{aligned}
$$

Obviously, if we restrict ourselves to the case of model given in (4), that is if we let $\theta_{1}=\theta_{2}=0$, expressions (26) and (27) lead to the same kind of equation for the dividends payment of the two classes of insurance business. In the same vein, if we consider the model given in (6), that is if we let $\theta_{0}=0$, Equations (22)-(23) and (26)-(27) are comparable.

\section{Boundary Conditions}

In this section we compute some boundary conditions satisfied by the net single premium $A(u, b)$ and $A_{i}\left(u_{i}, b_{i}\right)$ (see (12), (13) and (16), (17)) and by the discounted value of dividend payments $D(u, b)$ and $D_{i}\left(u_{i}, b_{i}\right)$ (see (22), (23) and (26), (27)), which will be used in Section 7 to provide some explicit solutions.

By simply substituting $u=0$ in Equation (13) we get:

$$
\left.\frac{\partial A(u, b)}{\partial u}\right|_{u=0}=\frac{1}{c}\left(\delta A(0, b)-\sum_{i=1}^{2}\left(\theta_{i}+\theta_{0}\right) \mu_{i}\right)
$$

whereas, by substituting $u=b$ in Equations (12) and (13), and combining them we obtain:

$$
\left.\frac{\partial A(u, b)}{\partial u}\right|_{u=b}=0
$$

In the same way we get the boundary conditions for $A_{i}\left(u_{i}, b_{i}\right), i=1,2$ :

$$
\begin{aligned}
& \left.\frac{\partial A_{i}\left(u_{i}, b_{i}\right)}{\partial u_{i}}\right|_{u_{i}=0}=\frac{1}{c_{i}}\left(\delta A_{i}\left(0, b_{i}\right)-\left(\theta_{i}+\theta_{0}\right) \mu_{i}\right) \\
& \left.\frac{\partial A_{i}\left(u_{i}, b_{i}\right)}{\partial u_{i}}\right|_{u_{i}=b_{i}}=0
\end{aligned}
$$

Analogously, by simply substituting $u=0$ in Equation (23) we get:

$$
\left.\frac{\partial D(u, b)}{\partial u}\right|_{u=0}=\frac{\delta}{c} D(0, b)
$$

whereas, by substituting $u=b$ in Equations (22) and (23), and combining them we obtain:

$$
\left.\frac{\partial D(u, b)}{\partial u}\right|_{u=b}=1
$$

In the same way we get the boundary conditions for $D_{i}\left(u_{i}, b_{i}\right), i=1,2$ : 


$$
\begin{aligned}
& \left.\frac{\partial D_{i}\left(u_{i}, b_{i}\right)}{\partial u_{i}}\right|_{u_{i}=0}=\frac{\delta}{c_{i}} D_{i}\left(0, b_{i}\right) \\
& \left.\frac{\partial D_{i}\left(u_{i}, b_{i}\right)}{\partial u_{i}}\right|_{u_{i}=b_{i}}=1
\end{aligned}
$$

\section{Explicit Solutions for Some Special Cases}

In this section we provide explicit solutions for the net single premium and the discounted value of dividend payments in some special cases.

We first consider the net single premium $A(u, b)$ and the discounted value of dividend payments $D(u, b)$. In the special case $\theta_{0}=0$ and $F_{i}(x)=1-\mathrm{e}^{-\beta x}$, with $\beta>0, i=1,2$, the integro-differential Equation (13) becomes:

$$
\begin{aligned}
& c \frac{\partial A(u, b)}{\partial u}-\left(\theta_{1}+\theta_{2}+\delta\right) A(u, b)+\frac{1}{\beta}\left(\theta_{1}+\theta_{2}\right) \\
& +A(0, b)\left(\theta_{1}+\theta_{2}\right) \mathrm{e}^{-\beta u}-u\left(\theta_{1}+\theta_{2}\right) \\
& =-\left(\theta_{1}+\theta_{2}\right) \mathrm{e}^{-\beta u} \int_{0}^{u} \beta(A(y, b)+y) \mathrm{e}^{\beta y} \mathrm{~d} y
\end{aligned}
$$

By deriving the previous equation with respect to $u$ and with some computations, we get the following second order linear homogeneous differential equation with constant coefficients:

$$
c \frac{\partial^{2} A(u, b)}{\partial u^{2}}-\left(\theta_{1}+\theta_{2}+\delta-\beta c\right) \frac{\partial A(u, b)}{\partial u}-\beta \delta A(u, b)=0
$$

whose characteristic equation is given by:

$$
c \lambda^{2}-\left(\theta_{1}+\theta_{2}+\delta-\beta c\right) \lambda-\beta \delta=0
$$

with distinct real solutions $\lambda_{1}<0<\lambda_{2}$. Using the boundary condition (28) and (29), we get the explicit solution of the net single premium $A(u, b)$ :

$$
A(u, b)=\frac{\theta_{1}+\theta_{2}}{\beta} \frac{\lambda_{2} \mathrm{e}^{\lambda_{2} b} \mathrm{e}^{\lambda_{1} u}-\lambda_{1} \mathrm{e}^{\lambda_{1} b} \mathrm{e}^{\lambda_{2} u}}{\left(\delta-c \lambda_{1}\right) \lambda_{2} \mathrm{e}^{\lambda_{2} b}-\left(\delta-c \lambda_{2}\right) \lambda_{1} \mathrm{e}^{\lambda_{1} b}} .
$$

In the same way, using our hypotheses, the integro-differential Equation (23) can be rewritten as follows:

$$
\begin{aligned}
& c \frac{\partial D(u, b)}{\partial u}-\left(\theta_{1}+\theta_{2}+\delta\right) D(u, b)+\left(\theta_{1}+\theta_{2}\right) D(0, b) \mathrm{e}^{-\beta u} \\
& =-\left(\theta_{1}+\theta_{2}\right) \mathrm{e}^{-\beta u} \int_{0}^{u} \beta D(y, b) \mathrm{e}^{\beta y} \mathrm{~d} y
\end{aligned}
$$

Therefore, using the boundary conditions (31) and (32), the explicit solution of the the discounted value of dividend payments $D(u, b)$ is given by:

$$
D(u, b)=\frac{\left(\delta-c \lambda_{2}\right) \mathrm{e}^{\lambda_{1} u}-\left(\delta-c \lambda_{1}\right) \mathrm{e}^{\lambda_{2} u}}{\left(\delta-c \lambda_{2}\right) \lambda_{1} \mathrm{e}^{\lambda_{1} b}-\left(\delta-c \lambda_{1}\right) \lambda_{2} \mathrm{e}^{\lambda_{2} b}},
$$

where $\lambda_{1}<0<\lambda_{2}$ are the two real distinct solutions of (34).

Now, we consider the net single premium $A_{i}\left(u_{i}, b_{i}\right)$ and the discounted value of dividend payments $D_{i}\left(u_{i}, b_{i}\right)$, with $i=1,2$. In the special case 
$F_{i}(x)=1-\mathrm{e}^{-\beta_{i} x}$, with $\beta_{i}>0, i=1,2$, the integro-differential Equation (17) becomes:

$$
\begin{aligned}
& c_{i} \frac{\partial A_{i}\left(u_{i}, b_{i}\right)}{\partial u_{i}}-\left(\theta_{0}+\theta_{i}+\delta\right) A_{i}\left(u_{i}, b_{i}\right)+\frac{1}{\beta_{i}}\left(\theta_{0}+\theta_{i}\right) \\
& +\left(\theta_{0}+\theta_{i}\right) A_{i}\left(0, b_{i}\right) \mathrm{e}^{-\beta_{i} u_{i}}-u_{i}\left(\theta_{0}+\theta_{i}\right) \\
& =-\left(\theta_{0}+\theta_{i}\right) \mathrm{e}^{-\beta_{i} u_{i}} \int_{0}^{u_{i}} \beta_{i}\left(A_{i}\left(y, b_{i}\right)+y\right) \mathrm{e}^{\beta_{i} y} \mathrm{~d} y, i=1,2
\end{aligned}
$$

By deriving the previous equation with respect to $u_{i}$ and with some computations, we get the following second order linear homogeneous differential equation with constant coefficients:

$$
c_{i} \frac{\partial^{2} A_{i}\left(u_{i}, b_{i}\right)}{\partial u_{i}^{2}}-\left(\theta_{0}+\theta_{i}+\delta-\beta_{i} c_{i}\right) \frac{\partial A_{i}\left(u_{i}, b_{i}\right)}{\partial u_{i}}-\beta_{i} \delta A_{i}(u, b)=0
$$

whose characteristic equation is given by:

$$
c_{i} \lambda^{2}-\left(\theta_{0}+\theta_{i}+\delta-\beta_{i} c_{i}\right) \lambda-\beta_{i} \delta=0,
$$

with distinct real solutions $\lambda_{1}<0<\lambda_{2}$. Using the boundary conditions (30), we get the explicit solution of the net single premium $A_{i}\left(u_{i}, b_{i}\right)$ :

$$
A_{i}\left(u_{i}, b_{i}\right)=\frac{\theta_{0}+\theta_{i}}{\beta_{i}} \frac{\lambda_{2} \mathrm{e}^{\lambda_{2} b_{i}} \mathrm{e}^{\lambda_{1} u_{i}}-\lambda_{1} \mathrm{e}^{\lambda_{1} b_{i}} \mathrm{e}^{\lambda_{2} u_{i}}}{\left(\delta-c_{i} \lambda_{1}\right) \lambda_{2} \mathrm{e}^{\lambda_{2} b_{i}}-\left(\delta-c_{i} \lambda_{2}\right) \lambda_{1} \mathrm{e}^{\lambda_{1} b_{i}}} .
$$

In the same way, using our hypotheses, the integro-differential Equation (27) can be rewritten as follows:

$$
\begin{aligned}
& c_{i} \frac{\partial D_{i}\left(u_{i}, b_{i}\right)}{\partial u_{i}}-\left(\theta_{0}+\theta_{i}+\delta\right) D_{i}\left(u_{i}, b_{i}\right)+\left(\theta_{0}+\theta_{i}\right) D_{i}\left(0, b_{i}\right) \mathrm{e}^{-\beta_{i} u_{i}} \\
& =-\left(\theta_{0}+\theta_{i}\right) \mathrm{e}^{-\beta_{i} u_{i}} \int_{0}^{u_{i}} \beta_{i} D_{i}\left(y, b_{i}\right) \mathrm{e}^{\beta_{i} y} \mathrm{~d} y, i=1,2
\end{aligned}
$$

Therefore, using the boundary conditions (33), the explicit solution of the the discounted value of dividend payments $D_{i}\left(u_{i}, b_{i}\right)$ is given by:

$$
D_{i}\left(u_{i}, b_{i}\right)=\frac{\left(\delta-c_{i} \lambda_{2}\right) \mathrm{e}^{\lambda_{1} u_{i}}-\left(\delta-c_{i} \lambda_{1}\right) \mathrm{e}^{\lambda_{2} u_{i}}}{\left(\delta-c_{i} \lambda_{2}\right) \lambda_{1} \mathrm{e}^{\lambda_{1} b_{i}}-\left(\delta-c_{i} \lambda_{1}\right) \lambda_{2} \mathrm{e}^{\lambda_{2} b_{i}}},
$$

where $\lambda_{1}<0<\lambda_{2}$ are the two real distinct solutions of (37).

\section{Final Conclusions}

In this paper we presented a two-dimensional risk model which is rather general with respect to the claim number processes, and we derived three different kinds of models which we studied in details. We assumed that the insurer applies a strategy of dividends payments and that he avoids the ruin through a dynamic solvency insurance. We considered two classical definitions of time of ruin, well-known in the literature. We obtained the integral and integro-differential equations for the net single premium of dynamic solvency insurance and for the discounted value of dividends payments. Further, we determined some boundary conditions, which allowed us to get explicit solutions for some special cases. The two-dimensional risk models have a lot of potential applications and have 
been, until now, intensively studied mainly in the ruin probability framework. The main contribution of our paper is the introduction of the dividends payments and the dynamic solvency insurance in the two-dimensional models; we also investigated the consequences of the proposed model under different assumptions. The two-dimensional models open the doors to various future developments, among which we consider of special interest the introduction of various kinds of reinsurance and the research of optimal reinsurance strategies under different objective functions, such as the minimization of the ruin probability or the maximization of the expected utility of terminal wealth. As some authors have already pointed out, the two-dimensional model can be extended to more than two classes of insurance business; therefore, a natural development of our study could be the use of the dividends, the dynamic solvency insurance and the reinsurance in these extended models.

\section{Acknowledgements}

We thank the Editor and the Referees for their comments.

\section{Conflicts of Interest}

The authors declare no conflicts of interest regarding the publication of this paper.

\section{References}

[1] Chan, W.-S., Yang H. and Zhang, L. (2003) Some Results on Ruin Probabilities in a Two-Dimensional Risk Model. Insurance: Mathematics and Economics, 32, 345-358. https://doi.org/10.1016/S0167-6687(03)00115-X

[2] Cai, J. and Li, H. (2007) Dependence Properties and Bounds for Ruin Probabilities in Multivariate Compound Risk Models. Journal of Multivariate Analysis, 98, 757-773. https://doi.org/10.1016/j.jmva.2006.06.004

[3] Dang, L., Zhu, N. and Zhang, H. (2009) Survival Probability for a Two-Dimensional Risk Model. Insurance: Mathematics and Economics, 44, 491-496.

https://doi.org/10.1016/j.insmatheco.2009.02.001

[4] Badescu, A.L., Cheung, E.C.K. and Rabehasaina, L. (2011) A Two-Dimensional Risk Model with Proportional Reinsurance. Journal of Applied Probability, 48, 749-765. https://doi.org/10.1239/jap/1316796912

[5] Gerber, H.U. (1981) On the Probability of Ruin in the Presence of a Linear Dividend Barrier. Scandinavian Actuarial Journal, 1981, 105-115.

[6] Gerber, H. and Pafumi, G. (1998) Pricing Dynamic Solvency Insurance and Investment Fund Protection. Decisions in Economics and Finance, 21, 125-146. https://doi.org/10.1007/BF02735319

[7] Feller, W. (1966) An Introduction to Probability Theory and Its Applications. 2nd Edition, Vol. 2, Wiley \& Sons, New York.

[8] Sundt, B. and Teugels, J.L. (1995) Ruin Estimates under Interest Force. Insurance: Mathematics and Economics, 16, 7-22. https://doi.org/10.1016/0167-6687(94)00023-8

[9] Inouye, D.I., Yang, E., Allen, G.I. and Ravikumar, P. (2017) A Review of Multiva- 
riate Distributions for Count Data Derived from the Poisson Distribution. WIREs Computational Statistics, 9, e1398.

[10] Papoulis, A. (1965) Probability, Random Variables and Stochastic Processes. McGraw-Hill, New York. 